\title{
A Complex Systems Perspective on Neuroimaging Studies of Behaviour and its Disorders
}

Authors

Federico E. Turkheimer, ${ }_{1}$ Fernando E. Rosas, $2,3,4$, Ottavia Dipasquale, Daniel Martins$_{1}$, Erik D. Fagerholm 1 , Paul Expert 5 , Frantisek Vasa 1 , Louis-David Lord6, Robert Leech

\section{Affiliations}

${ }_{1}$ Department of Neuroimaging, Institute of Psychiatry, Psychology and Neuroscience, King's College London, London, UK;

${ }_{2}$ Centre for Psychedelic Research, Department of Brain Sciences, Imperial College London, London, UK;

${ }_{3}$ Data Science Institute, Imperial College London, London, UK;

${ }_{4}$ Centre for Complexity Science, Imperial College London, London, UK;

${ }_{5}$ Global Digital Health Unit, School of Public Health, Imperial College London, London, UK. 6School of Medicine, Jagiellonian University, Krakow, Poland.

Corresponding author:

Prof. Federico E. Turkheimer

Institute of Psychiatry, Psychology and Neuroscience, King's College London

Centre for Neuroimaging Sciences

London SE5 9RT

Tel: +44(o) 2032283051

Email: federico.turkheimer@kcl.ac.uk

\section{Running title: The Brain as a Complex System}




\begin{abstract}
The study of complex systems deals with emergent behaviour that arises as a result of nonlinear spatio-temporal interactions between a large number of components both within the system, as well as between the system and its environment. There is a strong case to be made that neural systems as well as their emergent behaviour and disorders, can be studied within the framework of complexity science. In particular, the field of neuroimaging has begun to apply both theoretical and experimental procedures originating in complexity science - usually in parallel with traditional methodologies. Here, we demonstrate that the use of such traditional models may distort the outcomes of neuroimaging experiments hence affecting their interpretability and raising questions about their reliability. Therefore, we argue in favor of adopting a complex systems-based methodology in the study of neuroimaging, alongside appropriate experimental paradigms, and with minimal influences from non-complex systems approaches. Our exposition includes a review of the fundamental mathematical concepts, combined with practical examples and a compilation of results from the literature.
\end{abstract}




\section{INTRODUCTION}

One of the central aims of contemporary neuroimaging is to unify the models of neural structure and function in order to shed light, not just on the evolutionary organization of the brain, but also on its output, emergent behaviour, as well as on its disorders. Intuitively, one would expect the aetiology of brain disorders to be mainly driven by few dominant anatomical regions. However, evidence accumulated in recent years has unravelled a different picture. In fact, most studies indicate that the functional and structural variance in the human brain-during normal functioning, development, ageing and disease-tends to be widely distributed and can hardly be attributed to a set of specific regions (1-3). These findings have impacted the paradigms used to interpret neuroimaging data, which are gradually evolving from phrenological perspectives-that aimed to find associations between the variance in the data and parsimonious sets of regions $($ see $(4,5)$ for two contrasting views on the subject) - to embrace distributed analyses (6) that map the data into regional regulatory networks. This latter approach has been borrowed from the rich literature of complexity science, whose main aim is to progress our understanding of the essential features of complex systems. These two perspectives currently co-exist in neuroimaging analyses, and their tools are used, sometimes interchangeably, on similar datasets or within the same study (7-9).

In this work, we combine a number of mathematical concepts with practical examples and observations from the literature to build a case in favor of the use of complexity science analytics to study the brain through neuroimaging techniques. We aim to demonstrate that if the brain is a complex system, and there is strong evidence that it is, then the use of traditional models and associated analytics may distort the outcomes of imaging experiments. This issue, we believe, is one of the key drivers behind the lack of robustness, replicability and, ultimately, poor interpretability of a significant portion of contemporary neuroimaging studies. As a solution, we suggest that when a model of complexity is adopted, the choice of concepts and tools should be embraced together with appropriate experimental designs. We illustrate our position via mathematical concepts combined with practical examples, and a compilation of results from the literature.

\section{The Brain as A Complex SySTEM}

While the exact definition of a complex system is still under debate (10), there is consensus on four properties that all systems characterised as "complex" necessarily share. These properties are as follows (11):

1. Multiplicity. A complex system is made up of a large number of components, each of which interacts with its neighbouring components in relatively simple ways. 
2. Non-linearity. The interactions between components are nonlinear, e.g. changes in the output are not always proportional to the corresponding changes of the input.

3. Self-Organisation. The system self-organises spontaneously, in the absence of any form of centralised control.

4. Emergence. The system exhibits emergent behaviour, i.e. the macroscopic behaviour cannot be understood purely in terms of the microscopic interactions (12). Rather, the

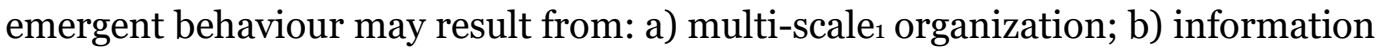
processing capability; c) dynamical spatiotemporal patterns; d) evolution.

Of these four properties, multiplicity and non-linearity can be seen as the fundamental enablers that - in the right circumstances - allow self-organisation and emergence to happen.

The rest of this article analyses the idea of the brain as a complex system, exploring each of these four properties under the light of recent evidence provided by contemporary neuroscience. It is to be noted that each of these properties has been studied in the neuroscience literature (14-18), but - to the best of our knowledge - there have been no unified accounts which look at the four of them with their interrelationships and consequences.

\section{From Phrenology to An Extended View of NeUroimaging Models}

Let us start considering the nature of the various parts that make the brain, which is related to the first property of complex systems. One of the longest-standing challenges in neuroscience is to decipher how variation in neural systems - both structural and functional - maps onto variation in behavioural phenotypes. The initial approach used in the analysis of imaging data was heavily influenced by a highly localized perspective relating neural and phenotypic variability (4). Its use stemmed from the success of neurology/neuropsychology at making differential diagnoses, relating dissociable cognitive/perceptual disorders to apparent localized damage. Even though the localized damage was just a partial view of the underlying pathology, it facilitated the easy intuition that there is a clear mapping between form and function. As a consequence, it focused on the use of mass univariate testing to map brain correlates of such phenotypic variation $(19,20)$. This paradigm triggered a long and enduring controversy, facing those with the view that it is paramount to protect results from false positives by using stringent significance thresholds (21), against those concerned about the loss of information due to high rates of false negatives (22). To a large extent, this discussion stems from conceptual differences in the a-priori expectation of the distribution of the brain's signals associated with a certain phenotype. Whereas the former position 
assumes that variability is highly concentrated in very few brain loci, the latter expects wider associations across brain networks.

Interestingly, this debate is reminiscent of a similar discussion that took place in the early 1900's amongst geneticists, who were divided between the Mendelians - those inspired by Mendel's work on pea genetics and saw phenotypes as the result of discrete, monogenic variants - and the biometricians, who did not accept that the inheritance of a continuous distribution of traits (e.g. height, intelligence, etc.) could be related to individual genes. At the time, R.A. Fisher resolved the issue by presenting his seminal work on the infinitesimal model (23), in which the inheritance of continuous traits could be explained by large numbers of variants, each of infinitesimal individual effect. In this theoretical work, Fisher conjectured that a large number of loci should be associated with what, at the time, were termed 'complex traits'. Only about 50 years have passed since the first genetic markers allowed for the detection of variants that have major effects, and only 20 years since singlenucleotide polymorphism technology provided dense markers throughout the genome. The combination of technological advancements, cost reductions, and increased sample sizes has allowed for the gradual expansion of models describing the genetic foundations of complex traits. It is now generally accepted that common single-nucleotide polymorphisms with small individual effect sizes - generally below the statistical significance levels of experimental designs - account for most of the variability of common genetic traits (24-26). Equally, complex disorders that are defined over a clinical spectrum demonstrate similarly broad genomic patterns (27). For example, recent work on the genetic architectures of schizophrenia has revealed that more than $70 \%$ of $1-\mathrm{Mb}$ genomic regions contain variants influencing the risk of the disorder (28). In fact, a more general 'omnigenic' view of complex traits and their heritability and evolution is now driving most of the genetic contemporary discourse $(29,30)$.

Moving from the genotype to the intermediate phenotype, there is increasing evidence that some of the remarkable computational capabilities of the brain, such as object recognition (still unmatched by machines) (31) - depend on the ability of neural systems to encode simple inputs into redundant widespread representations (31) and that this distributed architecture supports executive function (32). The conceptual models used in neuroimaging studies have evolved in the same direction. The field has been gradually shifting its focus from the analysis of to the analysis of covariance, e.g. moving from localized effects to distributed networks (33-37) However, a conscious, argumentative debate between these approaches has been lacking, and the two methods still co-exist. In particular, there remains the unanswered question of whether complex behaviour is supported by activity concentrated in few loci or by widely distributed networks. The latter view is supported by 
substantial evidence: Gonzalez-Castillo and colleagues used very large sample sizes or very extended single subject designs to reveal brain-wide functional support for simple tasks in fMRI studies $(38,39)$. Similarly, Haxby and colleagues (40) demonstrated that the representations of faces and objects in ventral temporal cortex are widely distributed and overlapping and second, using structural data, Chu et al (41) demonstrated that feature selection (e.g. regional variation) was as effective as total brain volume at predicting the progression from minimally cognitively impaired cohorts to Alzheimer's disease.

A complex system perspective on neural function results in predictions for the distribution of measured neural activity across the brain, which differs from predictions due to the localized perspective. For discussing some of these predictions, here we consider fMRI data from the Individual Brain Charting (IBC) project (42), which consists of a fMRI-curated dataset, acquired in a fixed environment from a permanent cohort of the same 12 participants during the performance of a dozen tasks, encompassing a wide range of motor and psychological domains. The results are presented in Figure 1, following the format in (29) where the zscores obtained from the testing of each task-vs-control contrast (only positive z-scores considered) were sorted in descending order and plotted versus the log of their rank. Figure $1 \mathrm{~A}$ and $1 \mathrm{~B}$ depict simpler (mostly motor) and higher mental processing tasks, respectively.

Figure 1: The panels illustrate the vectors of z-scores extracted from task-vs-baseline contrast (positive values only) maps obtained from fMRI studies of simple/motor tasks (1A) or more complex activities $(1 B)$. The $x$-axis represents the logarithm of the z-score ranks. The red lines represent the same $\mathrm{z}$-scores for the normal null distribution. The blue lines represent the James-Stein boundaries, i.e. the minimal decay of a z-score distribution that would justify the use of statistical thresholds for the estimation of the $z$-scored vector for each contrast (see Eq. [1]). The boundary curve has been arbitrarily scaled to the maximum $z$-score value for the group of tasks for which the theory suggests the use

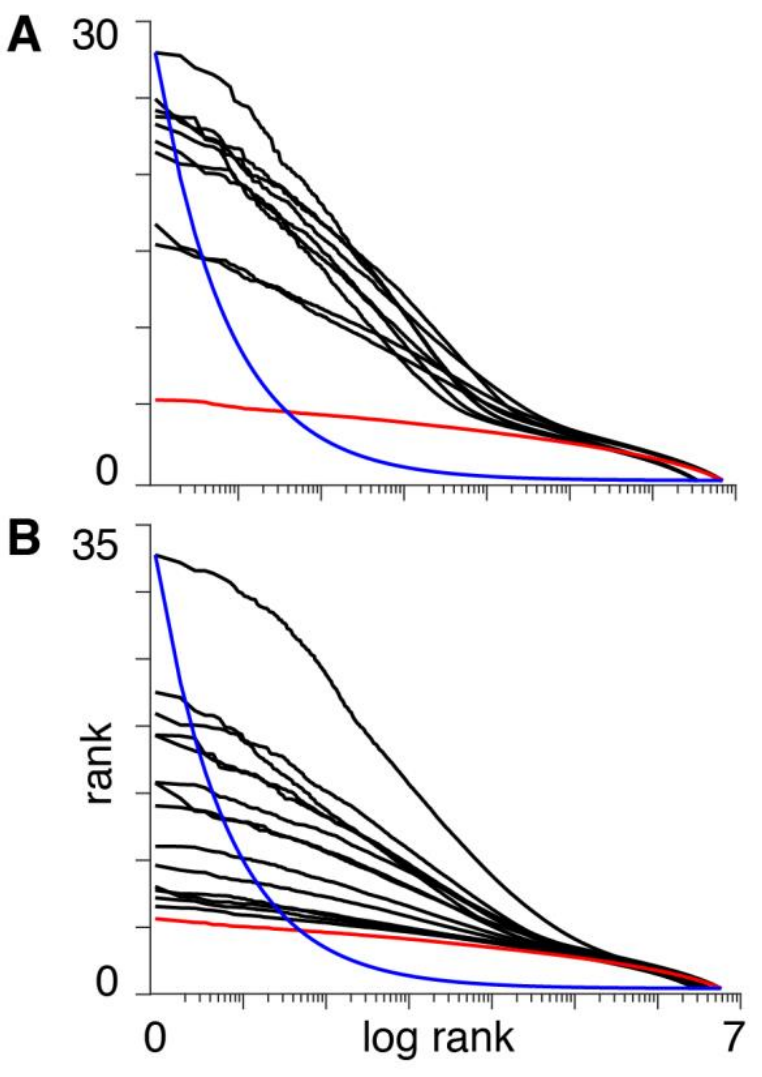
of shrinkage estimators for all vectors with smoother decays. 
These plots are to be contrasted with the expected null distribution (red) and the decay (blue):

$$
z_{k}=C k^{-1 / 2}
$$

where $z_{k}$ represents the z-score of rank $k$; $C$ is an arbitrary constant (which in the two plots in Figure 1 was fitted to the max z-score in each panel); and the exponent $-1 / 2$ is a minimum bound on the rate of decay of the z-scored vector - the estimation of which warrants the use of a statistical threshold, whereas vectors with a slower rate of decay require approaches based on weighted averages of the whole distribution $(43,44)$.

It may be seen that the curves in Figure 1 decay in a smooth manner - even in the simpler tasks, in which one would expect the signals to be described by a small number of coefficients with steeper decay rates - and do not satisfy the minimum requirement for the use of thresholding. In other words, the boundary in [3] is a way of formally stating that the use of thresholds to separate signal from noise in a multivariate vector is unwarranted when no such clear separation exists. In fact, any small change in experimental setting, imaging technology, pre-processing, as well as inter-individual variability (both neural activation, morphological or non-neural) could easily swap the ranks of the z-scores, thus limiting the reproducibility of the results (43). Furthermore, these plots confirm that the activity supporting the execution of a very large variety of tasks is distributed across much if not the entire neural system and that the brain therefore satisfies the first requirement in being classified as a complex system.

\section{INTERACTION AND NONLINEARITY}

Let us consider now non-linearity, the second key aspect of complexity. Nonlinearities within a complex system arise as a result of interactions between its elements. The brain offers several examples of nonlinear relationships between its physiological parameters. For example, there is the way in which V1 neurons respond to inputs (45) - where object recognition is performed by nonlinear operations on distributed, largely redundant representations (45) - or the response of evoked deep tissue pain (46) - where the response is linearly related to pain intensity in certain regions, but is nonlinear in others.

However, the point we want to make in this section is that nonlinearities in complex systems are generated by the interactions amongst elements when changes are applied to the organic whole. To illustrate this perspective, let's start with a simple theoretical illustration, which will then showcase how these inferences might apply to a real scenario. As a simple example, 
let us consider a population of rabbits that reproduce regularly with a certain birth-rate $R$. The relationship between the number of rabbits at time $t$, denoted by $N_{t}$, and the corresponding quantity at time $t+1$ can be expressed as

$$
N_{t+1}=R N_{t} \text {. }
$$

In particular, if one considers the case $R=2$ and runs three iterations of the model, starting from one rabbit at $t=O$ we obtain eight rabbits at $t=3$, while if we start with 2 rabbits the final total is sixteen. This confirms that this system is linear and that the totality of the system is equal to the sum of the parts, as two rabbits produce twice the offspring of a single one.

The linear model above can be readily turned into a nonlinear complex system with interacting elements if we introduce a constraint wherein offspring need to compete for resource. Then, the model in (2) turns into the simple, but effective Verhulst model (47):

$$
N_{t+1}=R\left(N_{t}-\frac{N_{t}^{2}}{P_{\max }}\right),
$$

where $P_{\max }$ represents the maximum capacity of the environment to keep the offspring alive. Setting $P_{\max }=10$, we run 3 iterations of this algorithm with an initial state of one rabbit to obtain four offspring. Running the same model with three iterations and starting with two rabbits we obtain five offspring. In this case, the offspring of two rabbits is not five-times the off-spring of one rabbit (see example illustrated in Figure 2).

Figure 2: The panel above illustrates a simple linear growth model whereas the offspring of two rabbits is always twice the number of offspring of a single rabbit, whatever the number of iterations.

Growth Model: $N_{t+1}=R^{*} N_{t}$

In the logistic model below, a resource constraint makes the rabbits compete for resources and, this time, the offspring of two rabbits is not twice the offspring of
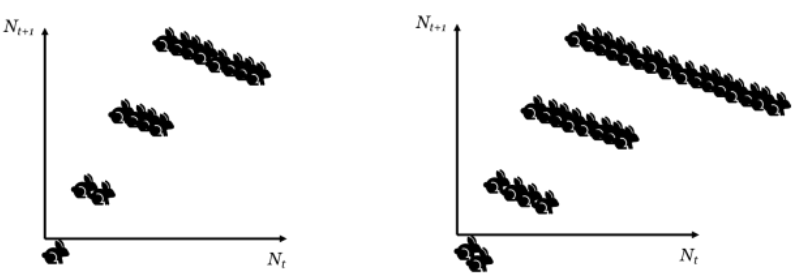

Logistic Model: $N_{t+1}=R *\left(N_{t}-N_{t}^{2} / \operatorname{Max} P\right)$ one
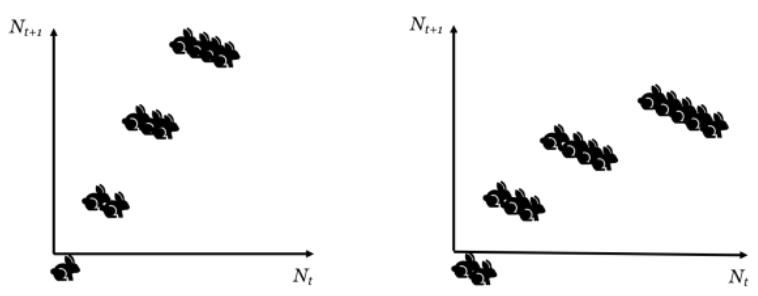
The above example demonstrates three important phenomena. Firstly, a simple resource constraint on the system introduces a highly non-trivial interaction between elements, so that the totality is not anymore equal to the sum of the parts. Secondly, this interaction translates into a non-linear behaviour observed within the system. Thirdly, the reproduction rate in (3) depends on the initial number of rabbits $N$.

The insights developed over the simple example can be used to understand more complicated issues related to brain development, brain ageing, and their disorders. For example, it has proven difficult for neuroimaging studies in autism to replicate both structural and functional results $(48,49)$, whereas the most consistent finding is the heterogeneity of imaging measures (50). One particularly puzzling finding is related to brain development of subjects within the autistic spectrum: these populations exhibit increased brain volumes in young children, whereas the rate of growth decreases subsequently during adolescence to meet the normal developmental curve between 10 and 15 years of age, with the volume of many structures finally declining atypically into adulthood (51-53). These findings are paradoxical from the perspective of a linear system; however, it is likely that a suitable nonlinear model of growth could explain why a more numerous initial state would experience a strong bias downwards in its developmental rate, especially when the whole system is placed under exogeneous pressure.

More generally, it is often noticeable in neuroimaging that individual developmental trajectories seem to provide much better resolution to categorize psychiatric disorders than cross-sectional data (54). This phenomenon is known in the literature of complexity science and dynamical systems as non-ergodicity, which characterises dynamical systems in which the statistics computed over single trajectories are not necessarily equal to the statistics across the population (55). This is equivalent to say that trajectories that start from different initial conditions may exhibit radically different dynamics. Non-ergodicity has dramatic consequences in the context of ageing and age-related disorders, where imaging studies have shown that cross-sectional analyses produce findings that are often in discrepancy with longitudinal analyses on the same subjects (56). In particular, longitudinal analyses unveil complex nonlinear trajectories of structural and functional data of anatomically parcellated regions, which can have radically different dynamics challenging most available explanatory models (57-60). Notably, during normal ageing, some brain areas may exhibit little to know age effect on regional cerebral volume, while others show a complex, non-linear relationship with age. This is mirrored by the significant intra-subject variability of cognitive decline - 
see, for example, the findings of the Nun Study (61), in which cognitive domains within the same individual deteriorate at very different rates (62).

When it comes to connecting the intermediate phenotype (i.e., the brain) with environmental modifiers on the one hand and genetic factors on the other, recent analysis of very large datasets have demonstrated that individual variability of MRI measures across ageing is spread across a large number of regional networks, or modes - each associated with overlapping environmental factors and genes (63). Interestingly, the biological and genetic factors involved in ageing accrue over three fundamental processes: metabolism, stress (particularly its effects on cellular senescence), and immunity. However, each of these processes is in itself a complex outcome of interactions amongst multiple genes and environmental factors, such as nutrition, environmental/psychosocial stress, and infections $(64,65)$.

Motivated by the above evidence, we propose here that the complex developmental and neurodegenerative patterns observed in structural and functional data are the result of nonlinear interactions generated by systemic pressures, which stem from metabolic and immune challenges punctuated across times (66-68). For example, metabolism is a fundamental determinant of neural function, as the brain accounts for $\sim 2 \%$ of total body mass, yet burns $\sim 20 \%$ of its energy, which is obtained through oxidative glucose metabolism, thus rendering the brain in constant demand of oxygen supply from cerebral blood flow (69). In fact, the human brain is, from a metabolic perspective, a scalable version of a primate brain, as the energy budget of the whole brain per neuron is approximately fixed across species and brain sizes (70) and hence its development has been strictly controlled by metabolic constraints (71). Brain metabolism is mostly devoted to sustaining synaptic activity (72) and metabolic rates influence neural circuitry and activity patterns by exerting selective pressure toward metabolically efficient wiring patterns (73), grey/white matter segregation (74), neuronal morphology $(75)$, and neural codes $(76,77)$. The metabolic demands of neural circuitry render the system highly susceptible to mitochondrial impairments, oxidative stress and deficits in glucose metabolism and perfusion. Therefore, the resulting global metabolic pressure stemming from noxious metabolic events translates into complex, non-linear interactions amongst the system's constituents. Furthermore, imaging, as well as cognitive and behavioural outcomes are strongly dependent on the timing of events across the age$\operatorname{span}(78)$.

Following the same principles, we propose that immunity-based second processes, that are recurrently associated to either developmental or neurodegenerative disorders, may generate system-wide pressures that then translate into complex outcomes. In fact, either maternal infections during gestation, or activation of the innate or adaptive immune system caused by 
stressful events, trauma or infections across a lifespan, have demonstrated to be the cause of psychiatric or degenerative conditions (79-84). The exact mechanism by which the immune system mechanistically affects brain function is still undetermined. One model proposes that peripheral cytokine concentrations may cause leakages in the blood-brain barrier, with activation of the immune system and consequential interference in transmission and plasticity $(81,85,86)$. Alternatively, we have recently proposed an indirect model of peripheral versus central immunity activation, where the mild peripheral inflammation observed in subjects with psychiatric conditions causes stiffening of the endothelial layers. This in turn causes a reduced permeability of the blood brain barrier to the transmission of small molecules, which (in chronic cases) leads to disturbed homeostasis and reduced functional activity (87).

\section{SElf-Organization ANd Criticality}

The third key property of complex systems is self-organization. Self-organization takes place whereas some form of global order arises as a consequence of the local non-linear interactions between parts of an initially disordered system (88). Importantly, the organization should emerge in a spontaneous manner, provided energy is available, and not as a result of the actions of a centralized controller or an external guidance. Therefore, the self-structuring events are often triggered by seemingly random fluctuations, which are dynamically amplified by some form of feedback enabled by the underlying non-linearities. The resulting organization is wholly decentralized, being distributed over all the components of the system, making it robust and able to endure (i.e. self-repair) or recover from substantial perturbations (89). The process of self-organization of a dynamical system can be visualized as a trajectory in $n$-dimensional phase space (where $n$ is the number of its elements) that evolves towards equilibria that can be described in terms of limit cycles. The evolution of the system is constrained to remain on the limit cycles and this constraint implies some form of coordination between its constituent elements (90, 91). A substantial amount of evidence, based on both human and animal experiments, indicates the important role of neural plasticity in the maturation of perceptual and cognitive processes. In other words, neural structures underlying these functions require sensory input for their maturation and genetic instructions are not sufficient to specify neuronal connections with significant precision $(92,93)$.

Importantly, the brain (as many natural complex systems) displays macroscopic patterns of activity with spatial and/or temporal scale invariance. The latter is a property which results in dynamical systems, e.g. those exhibiting turbulent flow (94), evolving in such a way that (except at the spatial or temporal extremes) it is not possible to distinguish the scale at which 
the system is being observed. This self-similar (or "fractal") character suggests that the selforganizing process that is ongoing during the development of the central nervous system (CNS) drives the system towards the inflection point of a second-order phase transition (11, 88). Pier Bak used the sand pile model as an exploration of self-organized criticality (SOC) (95). A sand pile can be organized by a simple rule that sees the grains falling randomly on a grid and accumulating until they topple sideways once they have reached a certain height (96). Once the grid starts filling up, avalanches of all sizes start cascading with an overall size-probability distribution which demonstrates the characteristic power law behaviour observed in self-similar dynamical systems (Figure 3).
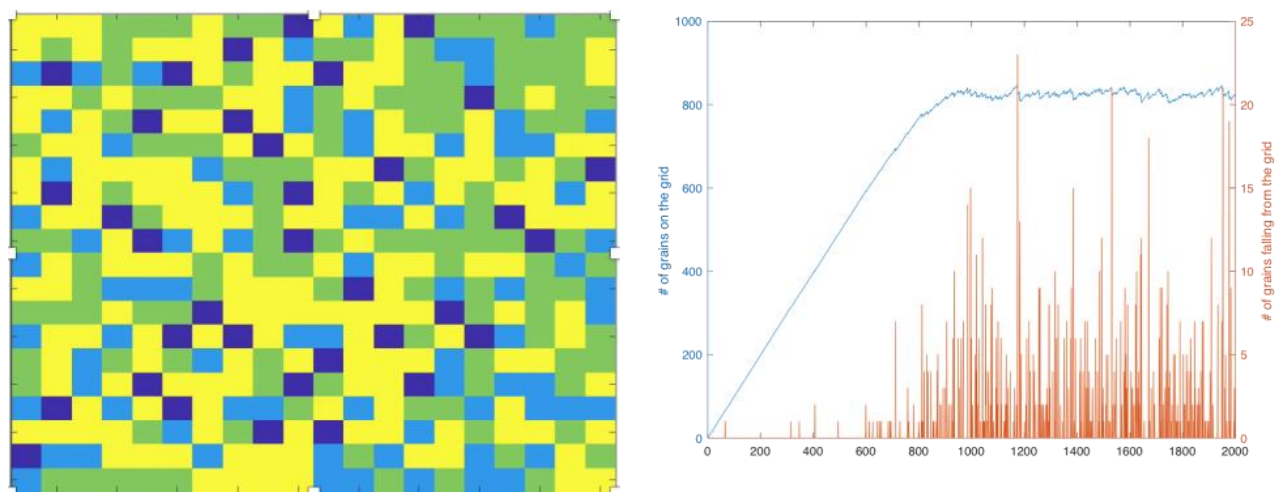

Figure 3: A simple sand-pile experiment simulated on a $20 x 20$ grid (left) whereas sand is dropped randomly on the square, and it topples sideways, as well off the grid, when a set limit for the height of a sand column is reached (4 grains in this case). The right panel shows the total sand in the grid through time, as well as the size of the avalanches falling off it.

Many natural systems tend towards such power law distributions, as these distributions allow for maximal adaptivity and other functional benefits (97). As in the case of the sand pile model, any perturbation to the system can be passed across scales and dissipated quickly - this endows the system with stability (98). Another example of this phenomenon is the energy cascade in turbulent hydrodynamic systems, in which kinetic energy is passed between eddies of decreasing size until it is distributed, at the smallest scales, to the viscosity of the liquid (99).

Other important requirement for self-organization is the abundance of available energy, in the same way that the sand pile needs a constant supply of grain to sustain itself in a critical state. Equally, the brain devotes up to $80 \%$ of its energy budget to maintaining its intrinsic activity (i.e. brain's activity in the absence of an overt motor, perceptual or cognitive task), whereas evoked activity in the brain does not use more than $1 \%$ of its metabolic reservoir (100, 101). This feature has been dubbed the Brain's Dark Energy (102). 
Self-organization rests upon four basic requirements (103): interactions between multiple constituent elements, strong non-linearity, possibly involving positive and negative feedback, a dynamic behavior striking a balance between exploitation and exploration, and availability of energy, which allows to overcome the natural tendency to increase entropy (e.g. disorder).

The above requirements can be readily observed in the organization of cortical structures. As summarized in (104), the brain (primarily the cortex) is made up of a multitude of elementary units, each of which consists of two neurons - a pyramidal excitatory unit coupled with a gamma aminobutyric acid (GABA) interneuron that, via a delayed negative feedback, transforms the firing of the unit into an oscillation in the gamma frequency (105), the so-called pyramidal interneuronal network gamma (PING) network. These elementary units are weakly connected via further GABA interneuron projections into and across the individual neural columns (106). Therefore, local oscillations in the gamma frequency are the binding force that generates rapid metastable dynamics, moving between synchronization and de-synchronization, which spread across the cortex. These elementary computational motifs have been observed across various brain regions, as well as across scales (104), reaching the long-range synchrony of neural activity. This supports the view of the brain as SOC system (107-110), with a distinctive fractal signature both in its structure and function $(104,111,112)$. The fractal distribution of neural output, also known as the $1 / f$ noise, can be observed in the behavioural repertoire, from the motor regions to the higher cognitive areas, capturing the range between basic physiological responses and creative abilities in the human brain $(113,114)$. Conversely, evidence suggests that this scaleinvariance in brain output may be lost during anaesthesia-induced loss of consciousness (115).

\section{EMERGENCE}

The fourth property of complex systems is that they are characterized by emergent behaviours. Complex systems, i.e. organisms or environments made up of very large numbers of elementary agents (e.g., ants in colonies, birds in a flock, or arrays of molecules or weather elements), can produce an impressive array of sophisticated behavior that cannot readily be explained solely by the physical properties of their constituent components. As a matter of fact, emergence - although still lacking a precise definition - has come to be recognized as a key ingredient of any system studied under the umbrella of complexity science (12). 
The concept of emergence is credited to the physiologist and philosopher George H. Lewes in his book Problems of Life and Mind (116). At the time, Lewes was concerned with reductionism, i.e. why all science doesn't ultimately reduce to physics. Victorian England in times of Lewes saw important advances in mechanics; however, chemical reactions (e.g. the production of water from oxygen and hydrogen) seemed to require explanations that were qualitative different from those that contemporary physics could provide. Building on this idea, Lewes drew a difference between mechanical effects and chemical effects-which he called "emergents". In his words:

“... The emergent is unlike its components in so far as these are incommensurable, and it cannot be reduced to their sum or their difference..." ((116), pp.413).

The notion of emergence was first brought into the neurosciences by Roger W Perry, the 1981 Nobel laureate for physiology and medicine, in the context of the mind-body problem. According to Perry, emergence “... is the idea, in brief, that conscious phenomena as emergent functional properties of brain processing exert an active control role as causal detents in shaping the flow patterns of cerebral excitation. Once generated from neural events, the higher order mental patterns and programs have their own subjective qualities and progress, operate and interact by their own causal laws and principles which are different from and cannot be reduced to those of neurophysiology." (117). In other words, the behaviour of the whole cannot be explained purely by focusing only on local spatiotemporal interactions between its elements in isolation- new theories are needed to explain these phenomena. Similarly, higher order cognitive function operates via laws that cannot be reduced to those of neurophysiology - instead they operate in the domain of psychology.

Despite their popularity, strong emergent approaches have been the source of numerous controversies. From a philosophical perspective, strong emergence has been accused of being logically inconsistent (118) and sustained on illegitimate metaphysics (119). Additionally, diverse concerns with respect to strongly emergent approaches in neuroscience have been raised, including arguments related to their plausibility in regard to empirical evidence (120), or to potential limits of their falsifiability (121). Finally, the fact that consciousness is usually the only proposed example of strong emergence in the natural world (see e.g. (122)) presumes an exceptionalism of consciousness that could be hard to justify. These difficulties are avoided by "weak emergent" approaches, which rely on computational models that start from biologically plausible elementary units and build higher order outputs, using tools and concepts borrowed by complexity science (12). These models are defined as "weakly" emergent, are not limited by ontological faults and have been proven to 
be scalable and able to realistically simulate the hierarchies of brain output and have now reached a level of maturity that enables predictions in the clinical realm $(104,120,123-127)$. More technically, weak emergence can be broadly categorised into computational irreducible and causal emergence approaches. The former, introduced by Bedau in (119) based on ideas from Wolfram (128), posits that emergence relates to limitations that hold in practice but not in principle; specifically, emergent properties would be generated by elements at microscopic levels in such complicated ways that they cannot be derived via explanatory shortcuts, but only by exhaustive simulation. While theoretically attractive, these approaches currently lack of ways of operationalizing their definitions, and hence their practical value is limited. In contrast, causal emergent approaches identify formal methods to assess the causal power exerted by macroscopic properties of a system. Causal emergence has been operationalized using Granger Causality (160), Pearl's do() calculus and effective information $(158,159)$, and most recently by Integrated Information Decomposition (162).

\section{THE TOOLS}

Having examined neural function in terms of the four main properties associated with complex systems, we now address the question of how the obtained insights can be operationalised.

Realising the large number of degrees of freedom that characterise the brain, one suggested approach is to shift the focus from the analysis of variance to the analysis of covariance. In other words, inference should be driven by the way the elements of the systems interact, and less so on how much they vary individually. The statistical literature provides numerous multivariate methods to study data covariance, which have been already been adapted to use in neuroimaging. A first approach involves using methods such as principal component analysis (PCA) (34), independent component analysis (ICA) (37), and partial least squares (33). Although not aimed at complexity analysis per se, these tools can be used to explore patterns in the data, as well as to derive measures of complexity. For example, Morgera Information Complexity is a measure of the extent to which covariance is distributed across principal components (129). A second approach is to represent covariance as networks, which are a popular tool to capture and explore covariance structures (6). Networks can be built on multiple metrics, ranging from standard correlation coefficients to mutual information or model-based indices that are generally better suited to capture non-linear interactions (130). Non-linear metrics are often preferred, as they avoid spurious patterns that can be caused by unjustified linear assumptions (131). Once the web of interactions has been modelled as vertices and edges, the topology of the structure can be further explored, both at the macro-scale level - using graph theoretical measures that summarize the network properties (35) - and at the meso-scale level where the topological stability of the 
structure can be further quantified $(132,133)$. A third approach to analyse covariant structures is to explore multivariate correlations, which capture high-order statistical properties that go beyond pairwise phenomena (134-137). High-order interactions can be used to build hypergraphs, which are networks were links can connect more than two nodes (138). Hypegraph analysis is an active area of ongoing research, which is opening novel avenues for neuroimaging research (see e.g. (139)).

Importantly, the brain is a non-linear system and, as such, its behaviour is dynamic. The discussions above have clarified the importance of experimental designs that move beyond stationary cross-sectional data paradigms and instead attempt, whenever practical, the acquisition of individual trajectories. This also enables the analysis of initial conditions, which are often relevant for complex systems; we have already shown how the artificial splitting of life-trajectories in development-adulthood-ageing may result in faulted inferences. The consideration of trajectories is also relevant at smaller timescales, including the duration of a BOLD MRI study where higher resting-state functional activity before a stimulus can result in less activation or more deactivation (140). It is often useful to characterise dynamics in terms of "states" or attractors, that is, regional patterns of activity between which the system oscillates (89). The number of these states and their stability fully define such dynamics and can be quantified using standard analytics (141-145) (see Figure 4) and have been used successfully in recent work on a broad spectrum of psychiatric disorders (146-152).

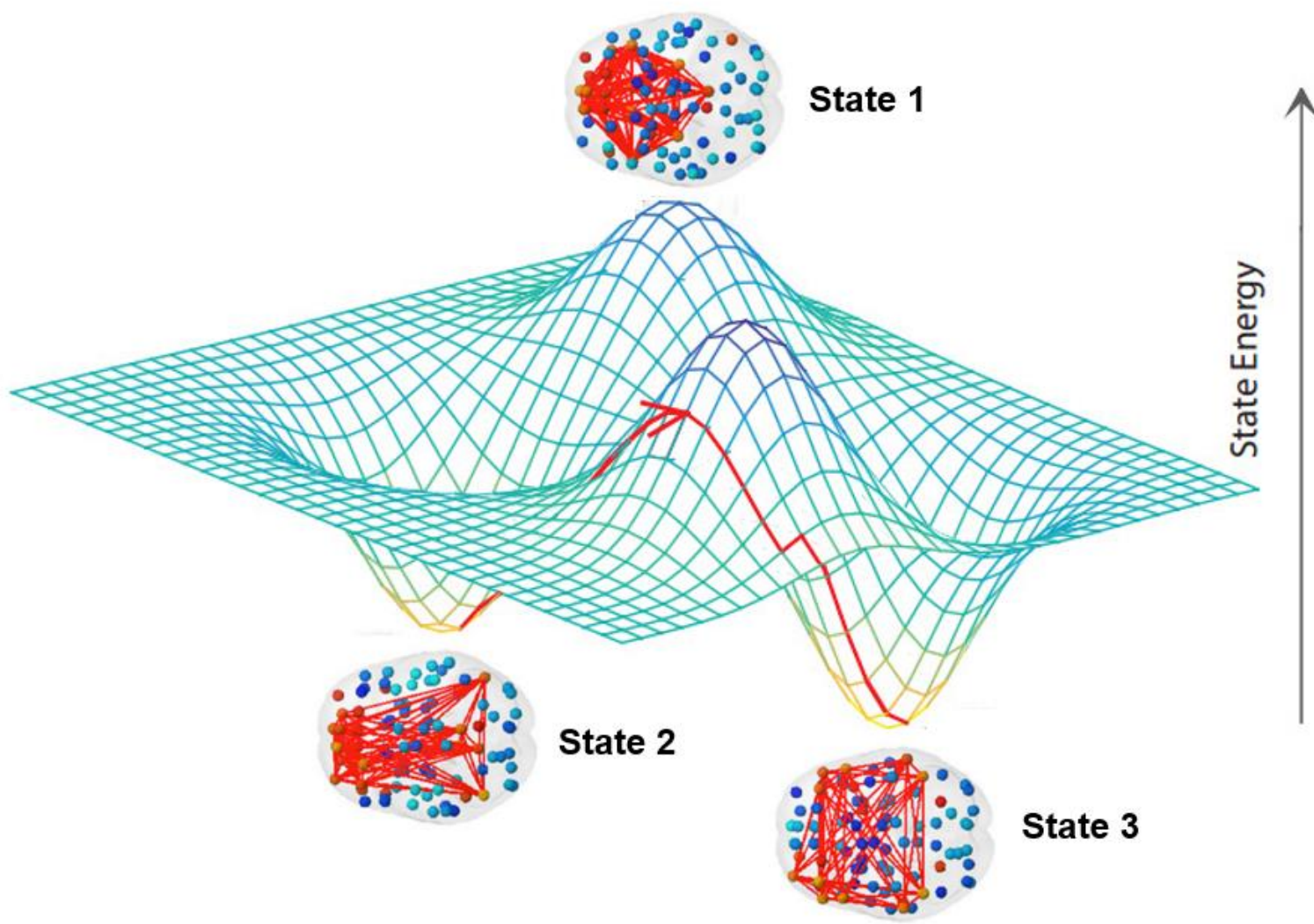


Figure 4: Brain states are more or less likely to be visited by the dynamics (red trace) over time by their relative energy/stability. Brain states are defined as intervals of temporal coherence (adapted from (153)).

Furthermore, if one accepts SOC as a model for brain, then the use of fractal geometry can be justified in studying brain structural data $(111,112)$ and its deviations from the SOC model. Fractal principles enable parsimonious methods to explain rich structures, holding great potential to explain brain structure and function. SOC approaches, however, face a number of important challenges (154), including the analysis of applicability of principles developed to study homogeneous systems (made e.g. by equivalent spins) over heterogeneous systems such as the brain. Also, there is a need to operationalize the beneficial properties of criticality, such as efficiency and robustness, so that they can render verifiable predictions. It must be clarified that these challenges do not put in question the core contribution of SOC into neuroscience, namely, that by neglecting the right details neuroscience might be capable of finding general underlying principles $(155,156)$.

Finally, the study of complex systems requires the quantification of two key properties; diversity and emergence. The first one can be described by a measure of entropy, for example Shannon's entropy (e.g. $-\sum_{i} p_{i} \log p_{i}$ where $p_{i}$ is the probability of state $i$ ), or variations of it including the Excess entropy $(157,158)$ and the entropy rate - this being closely related to the Lempel-Ziv complexity (159) and the statistical complexity of epsilon machines (160, 161). The second one, emergence, is a unique attribution of complex systems; the availability of one such measure would be a key tool in understanding the information flows between the various hierarchies/layers of models. So far, attempts to operationalize the various models of emergence have had limited applicability (162-165). This work is, however, fundamental to the progress of complexity science in general and it application to the neurosciences in particular, and novel modelling approaches and effective measures are appearing in the recent literature (166). These quantitative approaches allow researchers to frame hypotheses about emergence in a formal, rigorous manner, and test these hypotheses on fine-grained data-driven alternatives to traditional all-or-none classifications, opening a broad range of possibilities for applications.

\section{Conclusions}

We reviewed neural systems from the perspective of complexity science and matched the required properties of complex systems with evidence from contemporary neuroscience. The use of complexity science is not new to studies of the brain. However, its use has often been intertwined with standard analytical approaches that stem from older conceptual 
frameworks. What is now required is the acceptance of these new frameworks both in terms of experimentation and analysis. Complexity science is itself experiencing ongoing progress, and some of the required tools are perhaps yet to be discovered. Nonetheless, the potential insights to be gained by this paradigm are sufficiently promising to render this a very worthwhile endeavour. 


\section{REFERENCES}

1. Thompson PM, Vidal C, Giedd JN, Gochman P, Blumenthal J, Nicolson R, et al. Mapping adolescent brain change reveals dynamic wave of accelerated gray matter loss in very early-onset schizophrenia. Proc Natl Acad Sci U S A. 2001;98(20):11650-5.

2. Ecker C, Rocha-Rego V, Johnston P, Mourao-Miranda J, Marquand A, Daly EM, et al. Investigating the predictive value of whole-brain structural MR scans in autism: a pattern classification approach. Neuroimage. 2010;49(1):44-56.

3. Zhuo C, Li G, Lin X, Jiang D, Xu Y, Tian H, et al. The rise and fall of MRI studies in major depressive disorder. Transl Psychiatry. 2019;9(1):335.

4. Uttal WR. The new phrenology : the limits of localizing cognitive processes in the brain. Cambridge, Mass.: MIT Press; 2001. xviii, 255 p. p.

5. Donaldson DI. Parsing brain activity with fMRI and mixed designs: what kind of a state is neuroimaging in? Trends Neurosci. 2004;27(8):442-4.

6. Sporns O. Networks of the brain. Cambridge, Mass.: MIT Press; 2011. xi, 412 p., 8 p. of plates $\mathrm{p}$.

7. Pillet I, Op de Beeck H, Lee Masson H. A Comparison of Functional Networks Derived From Representational Similarity, Functional Connectivity, and Univariate Analyses. Front Neurosci. 2019;13:1348.

8. Brauchli C, Leipold S, Jancke L. Univariate and multivariate analyses of functional networks in absolute pitch. Neuroimage. 2019;189:241-7.

9. Hebart MN, Baker CI. Deconstructing multivariate decoding for the study of brain function. Neuroimage. 2018;180(Pt A):4-18.

10. Ladyman J, Lambert J, Wiesner K. What is a complex system? Euro Jnl Phil Sci. 2013;3.

11. Jensen HJ. Self-organized criticality : emergent complex behavior in physical and biological systems. Cambridge ; New York: Cambridge University Press; 1998. xiii, 153 p. p.

12. Turkheimer FE, Hellyer P, Kehagia AA, Expert P, Lord LD, Vohryzek J, et al. Conflicting emergences. Weak vs. strong emergence for the modelling of brain function. Neurosci Biobehav Rev. 2019;99:3-10.

13. Simon HA. The architecture of complexity. Proc Am Phil Soc. 1962;106:467-82.

14. Bassett DS, Gazzaniga MS. Understanding complexity in the human brain. Trends Cogn Sci. 2011;15(5):200-9.

15. Bullmore E, Barnes A, Bassett DS, Fornito A, Kitzbichler M, Meunier D, et al. Generic aspects of complexity in brain imaging data and other biological systems. Neuroimage. 2009;47(3):1125-34.

16. Deco G, Jirsa VK, McIntosh AR. Emerging concepts for the dynamical organization of resting-state activity in the brain. Nat Rev Neurosci. 2011;12(1):43-56.

17. Sporns O. The non-random brain: efficiency, economy, and complex dynamics. Front Comput Neurosci. 2011;5:5.

18. Telesford QK, Simpson SL, Burdette JH, Hayasaka S, Laurienti PJ. The brain as a complex system: using network science as a tool for understanding the brain. Brain Connect. 2011;1(4):295-308.

19. Friston KJ, Frith CD, Liddle PF, Frackowiak RS. Comparing functional (PET) images: the assessment of significant change. J Cereb Blood Flow Metab. 1991;11(4):690-9.

20. Worsley KJ, Marrett S, Neelin P, Vandal AC, Friston KJ, Evans AC. A unified statistical approach for determining significant signals in images of cerebral activation. Hum Brain Mapp. 1996;4(1):58-73. 
21. Eklund A, Nichols TE, Knutsson H. Cluster failure: Why fMRI inferences for spatial extent have inflated false-positive rates. Proc Natl Acad Sci U S A. 2016;113(28):79005.

22. Lieberman MD, Cunningham WA. Type I and Type II error concerns in fMRI research: re-balancing the scale. Soc Cogn Affect Neurosci. 2009;4(4):423-8.

23. Fisher RA. The correlation between relatives on the supposition of Mendelian inheritance. Trans R Soc Edinb. 1918;52:399-433.

24. Goldstein DB. Common genetic variation and human traits. N Engl J Med. 2009;360(17):1696-8.

25. Yang J, Benyamin B, McEvoy BP, Gordon S, Henders AK, Nyholt DR, et al. Common SNPs explain a large proportion of the heritability for human height. Nat Genet. 2010;42(7):565-9.

26. Shi H, Kichaev G, Pasaniuc B. Contrasting the Genetic Architecture of 30 Complex Traits from Summary Association Data. Am J Hum Genet. 2016;99(1):139-53.

27. Smoller JW, Andreassen OA, Edenberg HJ, Faraone SV, Glatt SJ, Kendler KS. Psychiatric genetics and the structure of psychopathology. Mol Psychiatry. 2019;24(3):409-20.

28. Loh PR, Bhatia G, Gusev A, Finucane HK, Bulik-Sullivan BK, Pollack SJ, et al. Contrasting genetic architectures of schizophrenia and other complex diseases using fast variance-components analysis. Nat Genet. 2015;47(12):1385-92.

29. Boyle EA, Li YI, Pritchard JK. An Expanded View of Complex Traits: From Polygenic to Omnigenic. Cell. 2017;169(7):1177-86.

30. Sella G, Barton NH. Thinking About the Evolution of Complex Traits in the Era of Genome-Wide Association Studies. Annu Rev Genomics Hum Genet. 2019;20:461-93.

31. DiCarlo JJ, Zoccolan D, Rust NC. How does the brain solve visual object recognition? Neuron. 2012;73(3):415-34.

32. Steinmetz NA, Zatka-Haas P, Carandini M, Harris KD. Distributed coding of choice, action and engagement across the mouse brain. Nature. 2019;576(7786):266-73.

33. McIntosh AR, Bookstein FL, Haxby JV, Grady CL. Spatial pattern analysis of functional brain images using partial least squares. Neuroimage. 1996;3(3 Pt 1):143-57.

34. Friston KJ, Frith CD, Liddle PF, Frackowiak RS. Functional connectivity: the principalcomponent analysis of large (PET) data sets. J Cereb Blood Flow Metab. 1993;13(1):514.

35. Bullmore E, Sporns O. Complex brain networks: graph theoretical analysis of structural and functional systems. Nat Rev Neurosci. 2009;10(3):186-98.

36. Greicius M. Resting-state functional connectivity in neuropsychiatric disorders. Curr Opin Neurol. 2008;21(4):424-30.

37. McKeown MJ, Makeig S, Brown GG, Jung TP, Kindermann SS, Bell AJ, et al. Analysis of fMRI data by blind separation into independent spatial components. Hum Brain Mapp. 1998;6(3):160-88.

38. Gonzalez-Castillo J, Hoy CW, Handwerker DA, Roopchansingh V, Inati SJ, Saad ZS, et al. Task Dependence, Tissue Specificity, and Spatial Distribution of Widespread Activations in Large Single-Subject Functional MRI Datasets at 7T. Cereb Cortex. 2015;25(12):4667-77.

39. Gonzalez-Castillo J, Saad ZS, Handwerker DA, Inati SJ, Brenowitz N, Bandettini PA. Whole-brain, time-locked activation with simple tasks revealed using massive averaging and model-free analysis. Proc Natl Acad Sci U S A. 2012;109(14):5487-92.

40. Haxby JV, Gobbini MI, Furey ML, Ishai A, Schouten JL, Pietrini P. Distributed and overlapping representations of faces and objects in ventral temporal cortex. Science. 2001;293(5539):2425-30. 
41. Chu C, Hsu AL, Chou KH, Bandettini P, Lin C, Alzheimer's Disease Neuroimaging I. Does feature selection improve classification accuracy? Impact of sample size and feature selection on classification using anatomical magnetic resonance images. Neuroimage. 2012;60(1):59-70.

42. Pinho AL, Amadon A, Ruest T, Fabre M, Dohmatob E, Denghien I, et al. Individual Brain Charting, a high-resolution fMRI dataset for cognitive mapping. Sci Data. 2018;5:180105.

43. Turkheimer FE, Aston JA, Banati RB, Riddell C, Cunningham VJ. A linear wavelet filter for parametric imaging with dynamic PET. IEEE Trans Med Imaging. 2003;22(3):289301.

44. Donoho DH, Johnstone IM. Minimax risk over lp -balls for lq -error. Probab Theory Related Fields. 1994;99:277-303.

45. Williams PE, Shapley RM. A dynamic nonlinearity and spatial phase specificity in macaque V1 neurons. J Neurosci. 2007;27(21):5706-18.

46. Loggia ML, Edwards RR, Kim J, Vangel MG, Wasan AD, Gollub RL, et al. Disentangling linear and nonlinear brain responses to evoked deep tissue pain. Pain. 2012;153(10):2140-51.

47. Verhulst P-F. Notice sur la loi que la population poursuit dans son accroisse- ment. Corresp Math Phys. 1838;10:113-21.

48. He Y, Byrge L, Kennedy DP. Nonreplication of functional connectivity differences in autism spectrum disorder across multiple sites and denoising strategies. Hum Brain Mapp. 2020;41(5):1334-50.

49. Lotspeich LJ, Kwon H, Schumann CM, Fryer SL, Goodlin-Jones BL, Buonocore MH, et al. Investigation of neuroanatomical differences between autism and Asperger syndrome. Arch Gen Psychiatry. 2004;61(3):291-8.

50. Lenroot RK, Yeung PK. Heterogeneity within Autism Spectrum Disorders: What have We Learned from Neuroimaging Studies? Front Hum Neurosci. 2013;7:733.

51. Wolff JJ, Jacob S, Elison JT. The journey to autism: Insights from neuroimaging studies of infants and toddlers. Dev Psychopathol. 2018;30(2):479-95.

52. Ha S, Sohn IJ, Kim N, Sim HJ, Cheon KA. Characteristics of Brains in Autism Spectrum Disorder: Structure, Function and Connectivity across the Lifespan. Exp Neurobiol. 2015;24(4):273-84.

53. Lange N, Travers BG, Bigler ED, Prigge MB, Froehlich AL, Nielsen JA, et al. Longitudinal volumetric brain changes in autism spectrum disorder ages 6-35 years. Autism Res. 2015;8(1):82-93.

54. Liberg B, Rahm C, Panayiotou A, Pantelis C. Brain change trajectories that differentiate the major psychoses. Eur J Clin Invest. 2016;46(7):658-74.

55. Griffith JS. On the stability of brain-like structures. Biophys J. 1963;3:299-308.

56. Nyberg L, Salami A, Andersson M, Eriksson J, Kalpouzos G, Kauppi K, et al. Longitudinal evidence for diminished frontal cortex function in aging. Proc Natl Acad Sci U S A. 2010;107(52):22682-6.

57. Fjell AM, Westlye LT, Grydeland H, Amlien I, Espeseth T, Reinvang I, et al. Critical ages in the life course of the adult brain: nonlinear subcortical aging. Neurobiol Aging. 2013;34(10):2239-47.

58. Good CD, Johnsrude IS, Ashburner J, Henson RN, Friston KJ, Frackowiak RS. A voxelbased morphometric study of ageing in 465 normal adult human brains. Neuroimage. 2001;14(1 Pt 1):21-36.

59. Fox NC, Schott JM. Imaging cerebral atrophy: normal ageing to Alzheimer's disease. Lancet. 2004;363(9406):392-4. 
60. Vinke EJ, de Groot M, Venkatraghavan V, Klein S, Niessen WJ, Ikram MA, et al. Trajectories of imaging markers in brain aging: the Rotterdam Study. Neurobiol Aging. 2018;71:32-40.

61. Snowdon DA, Nun S. Healthy aging and dementia: findings from the Nun Study. Ann Intern Med. 2003;139(5 Pt 2):450-4.

62. Salthouse TA. Trajectories of normal cognitive aging. Psychol Aging. 2019;34(1):17-24.

63. Smith SM, Elliott LT, Alfaro-Almagro F, McCarthy P, Nichols TE, Douaud G, et al. Brain aging comprises many modes of structural and functional change with distinct genetic and biophysical associations. Elife. 2020;9.

64. Shmookler Reis RJ, Bharill P, Tazearslan C, Ayyadevara S. Extreme-longevity mutations orchestrate silencing of multiple signaling pathways. Biochim Biophys Acta. 2009;1790(10):1075-83.

65. Kim SK. Common aging pathways in worms, flies, mice and humans. J Exp Biol. 2007;210(Pt 9):1607-12.

66. Lord LD, Allen P, Expert P, Howes O, Broome M, Lambiotte R, et al. Functional brain networks before the onset of psychosis: A prospective fMRI study with graph theoretical analysis. Neuroimage Clin. 2012;1(1):91-8.

67. Turkheimer FE, Selvaggi P, Mehta MA, Veronese M, Zelaya F, Dazzan P, et al. Normalizing the Abnormal: Do Antipsychotic Drugs Push the Cortex Into an Unsustainable Metabolic Envelope? Schizophr Bull. 2020;46(3):484-95.

68. Amad A, Expert P, Lord LD, Fovet T, Geoffroy PA. Plastic Adaptation to Pathology in Psychiatry: Are Patients with Psychiatric Disorders Pathological Experts? Neuroscientist. 2020;26(3):208-23.

69. Clarke DD, Sokoloff L. Regulation of Cerebral Metabolic Rate. In: Siegel GJ, Agranoff BW, Albers RW, editors. Basic Neurochemistry: Molecular, Cellular and Medical Aspects 6th edition. Philadelphia: Lippincott-Raven; 1999.

70. Herculano-Houzel S. Scaling of brain metabolism with a fixed energy budget per neuron: implications for neuronal activity, plasticity and evolution. PLoS One. 2011;6(3):e17514.

71. Fonseca-Azevedo K, Herculano-Houzel S. Metabolic constraint imposes tradeoff between body size and number of brain neurons in human evolution. Proc Natl Acad Sci U S A. 2012;109(45):18571-6.

72. Sibson NR, Dhankhar A, Mason GF, Rothman DL, Behar KL, Shulman RG. Stoichiometric coupling of brain glucose metabolism and glutamatergic neuronal activity. Proc Natl Acad Sci U S A. 1998;95(1):316-21.

73. Chen BL, Hall DH, Chklovskii DB. Wiring optimization can relate neuronal structure and function. Proc Natl Acad Sci U S A. 2006;103(12):4723-8.

74. Wen Q, Chklovskii DB. Segregation of the brain into gray and white matter: a design minimizing conduction delays. PLoS Comput Biol. 2005;1(7):e78.

75. Wen Q, Chklovskii DB. A cost-benefit analysis of neuronal morphology. J Neurophysiol. 2008;99(5):2320-8.

76. Levy WB, Baxter RA. Energy efficient neural codes. Neural Comput. 1996;8(3):531-43.

77. Balasubramanian V, Kimber D, Berry MJ, 2nd. Metabolically efficient information processing. Neural Comput. 2001;13(4):799-815.

78. Lord LD, Expert P, Huckins JF, Turkheimer FE. Cerebral energy metabolism and the brain's functional network architecture: an integrative review. J Cereb Blood Flow Metab. 2013;33(9):1347-54.

79. Gate D, Saligrama N, Leventhal O, Yang AC, Unger MS, Middeldorp J, et al. Clonally expanded CD8 T cells patrol the cerebrospinal fluid in Alzheimer's disease. Nature. 2020;577(7790):399-404. 
80. Labzin LI, Heneka MT, Latz E. Innate Immunity and Neurodegeneration. Annu Rev Med. 2018;69:437-49.

81. Miller AH, Raison CL. The role of inflammation in depression: from evolutionary imperative to modern treatment target. Nat Rev Immunol. 2016;16(1):22-34.

82. Meltzer A, Van de Water J. The Role of the Immune System in Autism Spectrum Disorder. Neuropsychopharmacology. 2017;42(1):284-98.

83. Meyer U. Neurodevelopmental Resilience and Susceptibility to Maternal Immune Activation. Trends Neurosci. 2019;42(11):793-806.

84. Richetto J, Chesters R, Cattaneo A, Labouesse MA, Gutierrez AMC, Wood TC, et al. Genome-Wide Transcriptional Profiling and Structural Magnetic Resonance Imaging in the Maternal Immune Activation Model of Neurodevelopmental Disorders. Cereb Cortex. 2017;27(6):3397-413.

85. Schedlowski M, Engler H, Grigoleit JS. Endotoxin-induced experimental systemic inflammation in humans: a model to disentangle immune-to-brain communication. Brain Behav Immun. 2014;35:1-8.

86. Dantzer R. Cytokine, sickness behavior, and depression. Immunol Allergy Clin North Am. 2009;29(2):247-64.

87. Turkheimer F, Althubaity N, Schubert J, Nettis MA, Cousins O, Dima D, et al. Increased serum peripheral C-reactive protein is associated with reduced small-molecule brain perfusion in healthy volunteers and subjects with major depressive disorder. 2020:2020.06.29.20138438.

88. Bak P. How nature works : the science of self-organized criticality. New York, NY, USA: Copernicus; 1996. xiii, 212 p., 8 p. of plates $p$.

89. Kelso JAS. Dynamic patterns : the self-organization of brain and behavior. Cambridge, Mass.: MIT Press; 1995. xvii, 334 p., 4 p. of plates p.

90. Ashby WR. Principles of the self-organizing dynamic system. J Gen Psychol. 1947;37(2):125-8.

91. Rosas FE, Mediano PAM, Ugarte M, Jensen HJ. An information-theoretic approach to self-organisation: Emergence of complex interdependencies in coupled dynamical systems. Entropy. 2018;20(10).

92. Singer W. The brain as a self-organizing system. Eur Arch Psychiatry Neurol Sci. 1986;236(1):4-9.

93. Tetzlaff C, Okujeni S, Egert U, Worgotter F, Butz M. Self-organized criticality in developing neuronal networks. PLoS Comput Biol. 2010;6(12):e1001013.

94. Bohr T. Dynamical systems approach to turbulence. Cambridge ; New York: Cambridge University Press; 1998. xx, 350 p. p.

95. Bak P, Paczuski M. Complexity, contingency, and criticality. Proc Natl Acad Sci U S A. 1995;92(15):6689-96.

96. Pruessner G. Self-organised criticality; theory, models and characterisation. Cambridge: Cambridge University Press; 2012.

97. Shew WL, Plenz D. The functional benefits of criticality in the cortex. Neuroscientist. 2013;19(1):88-100.

98. Bak P. Self-Organized Criticality: An Explanation of 1/f Noise. . Physical Review Letters 1987;59(4):381-4.

99. Kolmogorov AN. The local structure of isotropic turbulence in an incompressible viscous fluid. . Dokl Akad Nauk SSSR. 1941;30:301-5.

100. Raichle ME. The restless brain: how intrinsic activity organizes brain function. Philos Trans R Soc Lond B Biol Sci. 2015;370(1668).

101.Raichle ME, Mintun MA. Brain work and brain imaging. Annu Rev Neurosci. 2006;29:449-76. 
102.Raichle ME. Neuroscience. The brain's dark energy. Science. 2006;314(5803):1249-50.

103.NBonabeau E, Dorigo M, Theraulaz G. Swarm intelligence: from natural to artificial systems: OUP USA; 1999.

104.Turkheimer FE, Leech R, Expert P, Lord LD, Vernon AC. The brain's code and its canonical computational motifs. From sensory cortex to the default mode network: A multi-scale model of brain function in health and disease. Neurosci Biobehav Rev. 2015;55:211-22.

105.Borgers C, Kopell N. Synchronization in networks of excitatory and inhibitory neurons with sparse, random connectivity. Neural Comput. 2003;15(3):509-38.

106. Helmstaedter M, Sakmann B, Feldmeyer D. Neuronal correlates of local, lateral, and translaminar inhibition with reference to cortical columns. Cereb Cortex. 2009;19(4):926-37.

107.Cabral J, Luckhoo H, Woolrich M, Joensson M, Mohseni H, Baker A, et al. Exploring mechanisms of spontaneous functional connectivity in MEG: how delayed network interactions lead to structured amplitude envelopes of band-pass filtered oscillations. Neuroimage. 2014;90:423-35.

108.Cabral J, Hugues E, Sporns O, Deco G. Role of local network oscillations in resting-state functional connectivity. Neuroimage. 2011;57(1):130-9.

109. Roberts JA, Gollo LL, Abeysuriya RG, Roberts G, Mitchell PB, Woolrich MW, et al. Metastable brain waves. Nat Commun. 2019;10(1):1056.

110.Tognoli E, Kelso JA. The metastable brain. Neuron. 2014;81(1):35-48.

111.Squarcina L, De Luca A, Bellani M, Brambilla P, Turkheimer FE, Bertoldo A. Fractal analysis of MRI data for the characterization of patients with schizophrenia and bipolar disorder. Phys Med Biol. 2015;60(4):1697-716.

112.Expert P, Lambiotte R, Chialvo DR, Christensen K, Jensen HJ, Sharp DJ, et al. Selfsimilar correlation function in brain resting-state functional magnetic resonance imaging. J R Soc Interface. 2011;8(57):472-9.

113.Levitin DJ, Chordia P, Menon V. Musical rhythm spectra from Bach to Joplin obey a 1/f power law. Proc Natl Acad Sci U S A. 2012;109(10):3716-20.

114.Palva JM, Zhigalov A, Hirvonen J, Korhonen O, Linkenkaer-Hansen K, Palva S. Neuronal long-range temporal correlations and avalanche dynamics are correlated with behavioral scaling laws. Proc Natl Acad Sci U S A. 2013;110(9):3585-90.

115.Scott G, Fagerholm ED, Mutoh H, Leech R, Sharp DJ, Shew WL, et al. Voltage imaging of waking mouse cortex reveals emergence of critical neuronal dynamics. J Neurosci. 2014;34(50):16611-20.

116.Lewes GH. Problems of life and mind. Boston,: Houghton, Osgood and company; 1879.

117.Sperry RW. Mind-brain interaction: mentalism, yes; dualism, no. Neuroscience. 1980;5(2):195-206.

118. Bedau M. Downward causation and the autonomy of weak emergence. Principia: an international journal of epistemology. 2002;6(1):5-50.

119.Bedau M. Weak emergence. Noûs. 1997;31:375-99.

120.Turner R. Myelin and Modeling: Bootstrapping Cortical Microcircuits. Front Neural Circuits. 2019;13:34.

121.Colombo M, Wright C. First principles in the life sciences: the free-energy principle, organicism, and mechanism. . Synthese. 2018:1-26.

122.Chalmers DJ. Strong and weak emergence. In: Davies PCaP, editor. The re-emergence of emergence: Oxford University Press; 2006. p. 244-56.

123.Fagerholm ED, Hellyer PJ, Scott G, Leech R, Sharp DJ. Disconnection of network hubs and cognitive impairment after traumatic brain injury. Brain. 2015;138(Pt 6):1696-709. 
124.Zimmermann J, Perry A, Breakspear M, Schirner M, Sachdev P, Wen W, et al. Differentiation of Alzheimer's disease based on local and global parameters in personalized Virtual Brain models. Neuroimage Clin. 2018;19:240-51.

125.Proix T, Bartolomei F, Guye M, Jirsa VK. Individual brain structure and modelling predict seizure propagation. Brain. 2017;140(3):641-54.

126.Lord LD, Stevner AB, Deco G, Kringelbach ML. Understanding principles of integration and segregation using whole-brain computational connectomics: implications for neuropsychiatric disorders. Philos Trans A Math Phys Eng Sci. 2017;375(2096).

127.Deco G, Kringelbach ML. Great expectations: using whole-brain computational connectomics for understanding neuropsychiatric disorders. Neuron. 2014;84(5):892905.

128.Wolfram S. A new kind of science. Champaign, IL: Wolfram media; 2002.

129. Morgera SD. Information theoretic covariance complexity and its relation to pattern recognition. IEEE Transactions on Systems, Man, and Cybernetics 1985;15(5):608 - 19.

130. Song L, Langfelder P, Horvath S. Comparison of co-expression measures: mutual information, correlation, and model based indices. BMC Bioinformatics. 2012;13:328.

131.Bright MG, Murphy K. Is fMRI "noise" really noise? Resting state nuisance regressors remove variance with network structure. Neuroimage. 2015;114:158-69.

132.Petri G, Expert P, Turkheimer F, Carhart-Harris R, Nutt D, Hellyer PJ, et al. Homological scaffolds of brain functional networks. J R Soc Interface. 2014;11(101):20140873.

133.Expert P, Lord LD, Kringelbach ML, Petri G. Editorial: Topological Neuroscience. Netw Neurosci. 2019;3(3):653-5.

134.Mediano PAM, Rosas FE, Carhart-Harris R, Seth AK, Barrett AB. Beyond integrated information: a taxonomy of information dynamics phenomena. arXiv [Internet]. 2019.

135.Baudot P, Tapia M, Bennequin D, Goaillard J. Topological information data analysis. Entropy. 2019;21(9).

136.Rosas FE, Mediano PAM, Gastpar M, Jensen HJ. Quantifying high-order interdependencies via multivariate extensions of the mutual information. Phys Rev E. 2019;100(3-1):032305.

137.Tononi G, Sporns O, Edelman GM. A measure for brain complexity: relating functional segregation and integration in the nervous system. Proc Natl Acad Sci U S A. 1994;91(11):5033-7.

138. Bretto A. Hypergraph theory. An Introduction: Springer; 2013.

139. Gatica M, Cofré R, Mediano PAM, Rosas FE, Orio P, Diez I, et al. High-order interdependencies in the aging brain 2020.

140.He BJ. Spontaneous and task-evoked brain activity negatively interact. J Neurosci. 2013;33(11):4672-82.

141.Gonzalez-Castillo J, Bandettini PA. Task-based dynamic functional connectivity: Recent findings and open questions. Neuroimage. 2018;180(Pt B):526-33.

142.Calhoun VD, Miller R, Pearlson G, Adali T. The chronnectome: time-varying connectivity networks as the next frontier in fMRI data discovery. Neuron. 2014;84(2):262-74.

143. Hutchison RM, Womelsdorf T, Allen EA, Bandettini PA, Calhoun VD, Corbetta M, et al. Dynamic functional connectivity: promise, issues, and interpretations. Neuroimage. 2013;80:360-78.

144.Gu S, Betzel RF, Mattar MG, Cieslak M, Delio PR, Grafton ST, et al. Optimal trajectories of brain state transitions. Neuroimage. 2017;148:305-17. 
145.Lord LD, Expert P, Atasoy S, Roseman L, Rapuano K, Lambiotte R, et al. Dynamical exploration of the repertoire of brain networks at rest is modulated by psilocybin. Neuroimage. 2019;199:127-42.

146.de Lacy N, Calhoun VD. Dynamic connectivity and the effects of maturation in youth with attention deficit hyperactivity disorder. Netw Neurosci. 2019;3(1):195-216.

147.de Lacy N, Doherty D, King BH, Rachakonda S, Calhoun VD. Disruption to control network function correlates with altered dynamic connectivity in the wider autism spectrum. Neuroimage Clin. 2017;15:513-24.

148. Rabany L, Brocke S, Calhoun VD, Pittman B, Corbera S, Wexler BE, et al. Dynamic functional connectivity in schizophrenia and autism spectrum disorder: Convergence, divergence and classification. Neuroimage Clin. 2019;24:101966.

149.Kaiser RH, Whitfield-Gabrieli S, Dillon DG, Goer F, Beltzer M, Minkel J, et al. Dynamic Resting-State Functional Connectivity in Major Depression. Neuropsychopharmacology. 2016;41(7):1822-30.

150.Jin C, Jia H, Lanka P, Rangaprakash D, Li L, Liu T, et al. Dynamic brain connectivity is a better predictor of PTSD than static connectivity. Hum Brain Mapp. 2017;38(9):447996.

151. Sakoglu U, Pearlson GD, Kiehl KA, Wang YM, Michael AM, Calhoun VD. A method for evaluating dynamic functional network connectivity and task-modulation: application to schizophrenia. MAGMA. 2010;23(5-6):351-66.

152.Lurie DJ, Kessler D, Bassett DS, Betzel RF, Breakspear M, Kheilholz S, et al. Questions and controversies in the study of time-varying functional connectivity in resting fMRI. Netw Neurosci. 2020;4(1):30-69.

153.Gu S, Cieslak M, Baird B, Muldoon SF, Grafton ST, Pasqualetti F, et al. The Energy Landscape of Neurophysiological Activity Implicit in Brain Network Structure. Sci Rep. 2018;8(1):2507.

154.Beggs JM, Timme N. Being critical of criticality in the brain. Front Physiol. 2012;3:163.

155. Chialvo DR. Life at the edge: complexity and criticality in biological function. arXiv [Internet]. 2018.

156. Cofré R, Herzog R, Corcoran D, Rosas FE. A Comparison of the Maximum Entropy Principle Across Biological Spatial Scales. Entropy. 2019;21(10):1009.

157.Grassberger P. Toward a quantitative theory of self-generated complexity. International Journal of Theoretical Physics. 1986;25(9):907-38.

158. Crutchfield JP, Feldman DP. Regularities unseen, randomness observed: Levels of entropy convergence. . Chaos: An Interdisciplinary Journal of Nonlinear Science. 2003;13(1):25-54.

159.Schartner MM, Carhart-Harris RL, Barrett AB, Seth AK, Muthukumaraswamy SD. Increased spontaneous MEG signal diversity for psychoactive doses of ketamine, LSD and psilocybin. Sci Rep. 2017;7:46421.

160.Shalizi CR, Crutchfield JP. Computational mechanics: Pattern and prediction, structure and simplicity. Journal of statistical physics. 2001;104(3-4):817-79.

161.Muñoz RN, Leung A, Zecevik A, Pollock FA, Cohen D, van Swinderen B, et al. General anesthesia reduces complexity and temporal asymmetry of the informational structures derived from neural recordings in Drosophila. PHYSICAL REVIEW RESEARCH. 2020;2(2).

162.Hoel EP, Klein B. The emergence of informative higher scales in complex networks. Complexity. 2020;2020.

163. Hoel EP. When the map is better than the territory. Entropy. 2017;19.

164.Seth AK. Measuring autonomy and emergence via Granger causality. Artif Life. 2010;16(2):179-96. 
165.Hoel EP, Albantakis L, Tononi G. Quantifying causal emergence shows that macro can beat micro. Proc Natl Acad Sci U S A. 2013;110(49):19790-5.

166.Rosas FE, Mediano PAM, Jensen HJ, Seth AK, Barrett AB, Carhart-Harris R, et al. Reconciling emergences: An information-theoretic approach to identify causal emergence in multivariate data. arXiv>q-bio [Internet]. 2020. 